\title{
Beyond positive and negative trait affect: Flourishing through music engagement
}

\author{
TanChyuan Chin ${ }^{1 *}$ and Nikki S Rickard ${ }^{2}$
}

\author{
* Correspondence: \\ tanchyuan.chin@unimelb.edu.au \\ ${ }^{1}$ Melbourne Graduate School of \\ Education, The University of \\ Melbourne, 100 Leicester Street, \\ Parkville (3010), VIC, Australia \\ Full list of author information is \\ available at the end of the article
}

\begin{abstract}
Background: Mental health is not only the absence of mental illness, but also the presence of positive well-being. Music is often cited as an effective tool for regulating emotions and may also promote well-being because music facilitates one's ability to regulate the experience and expression of emotions.

Methods: This study aimed to examine the pathways by which music use for cognitive and emotional regulation purposes may predict mental health outcomes, as measured by self-reports of well-being and negative emotional states, while controlling for trait affect. The mediating effects of emotion regulation were examined in a diverse sample of 565 working adults (126 male, 439 females) between the ages of 18 and $68(M=24.00, S D=6.21)$.

Results: Results demonstrated that music engagement for the purposes of cognitive and emotional regulation predicts flourishing in life, through the mediation pathway of cognitive reappraisal, even after controlling for trait affect.

Conclusions: This study provides new evidence that purposeful music use for cognitive and emotional regulation may promote mental health beyond levels normally associated with positive consequences of balanced affective states.
\end{abstract}

The benefits of music are well documented in academic literature and popular media, with two recent volumes that bring together an array of studies on music, health and well-being (MacDonald et al., 2012; Rickard and McFerran, 2012). Of particular interest to this research is the use of music to promote mental health.

The World Health Organization defines mental health as "a state of well-being in which the individual realizes his or her own abilities, can cope with the normal stresses of life, can work productively and fruitfully, and is able to make a contribution to his or her community" (World Health Organization, 2005). Mental health is not only the absence of mental illness, but also the presence of positive well-being (Keyes, 2005; World Health Organization, 2005). The dual factor model conceptualizes mental illness and positive mental health as representing two related, but distinct dimensions (Antaramian et al., 2010; Keyes, 2005), and has been validated in both adolescent (Keyes, 2006; Suldo and Shaffer, 2008) and adult samples (Keyes et al., 2008; Westerhof and Keyes, 2010). According to the mental health continuum, individuals who are flourishing in life have high levels of well-being and are free from symptoms of mental illness (Keyes, 2002). In this continuum, it is also possible for individuals with mental illness to experience optimal mental health, and for individuals without mental illness to have minimal mental health (Keyes, 2002). A 10-year follow-up study of mental 
health and illness found that improvements in positive well-being predicted lower incidence of mental illness, and that declines in well-being predicted greater incidence of mental illness (Keyes et al., 2010). This suggests that states of mental health and mental illness are not static, and therefore, research that focuses on activities that promote well-being and reduce levels of mental illness should be a priority.

\section{The role of music in promoting mental health}

Music is a widely used leisure activity which has demonstrated a capacity to improve well-being (Bradt et al., 2011; MacDonald et al., 2012; Rickard and McFerran, 2012) and impact on symptoms of mental illness, such as depression and anxiety (Bradt and Dileo, 2009; Maratos et al., 2008; Siedliecki and Good, 2006). Music can be used to cope with life stressors and is not only cited as a personal mood management strategy (DeNora, 2000; Hays and Minichiello, 2005; Laukka, 2007; Saarikallio, 2007; Sloboda, 1999), but is also used frequently in hospital settings to reduce pain, anxiety or stress, and even to reduce the length of hospitalization (Standley, 1995). Music can also be used to improve task performance, from prolonging time spent on task and enhancing quality of work (Lesiuk, 2005) to mental preparation before a competitive event (Bishop et al., 2007; Karageorghis and Terry, 1997). Music also plays an important role in interpersonal relationships by facilitating the communication of feelings and identity (Hargreaves and North, 1997; MacDonald et al., 2002). Nonetheless, there is also evidence that everyday music use is associated with symptoms of psychopathology (Miranda and Claes, 2007, 2009; North and Hargreaves, 2006, 2008). It is therefore imperative to understand how different types of music use might explain its diverse relationships with well-being and symptoms of psychopathology, such as depression, anxiety and stress. This understanding will help inform the conditions under which the use of music to promote mental health can be confidently advocated.

\section{Measuring benefits of music engagement for mental health}

Positive mental health is a desirable outcome of music engagement. Whilst individuals engage with music using two broad processes, production (e.g., performing) and reception (e.g., listening), to date, most research on psychological benefits of music has tended to operationalize musicianship in terms of a specialized capacity to produce music (e.g., see Chin and Rickard, 2012a). This greatly limits our understanding of the effects of everyday music use in the general population, many of whom have little or no formal music training, but still actively engage with music for a variety of important reasons such as emotion regulation, constructing and strengthening of identity, creating and enhancing interpersonal relationships, relieving tension or stress, and getting through difficult times (DeNora, 2000; Laiho, 2004; North et al., 2000). For example, the goal or desire to improve one's mood can be achieved through a variety of music activities, such as dancing, listening, singing, or playing an instrument. Therefore, using a measure of music engagement which incorporates processes, functions and motivations of music use, provides the opportunity to evaluate mental health outcomes of everyday music use, instead of merely relying on the amount of formal music training that one had received. 


\section{Music use for cognitive and emotional regulation}

Music is often used for cognitive and emotional regulation purposes (Chamorro-Premuzic and Furnham, 2007; DeNora, 1999; North et al., 2000; Saarikallio, 2011; Saarikallio and Erkkilä, 2007) and has been rated as one of the most effective methods of mood regulation (Thayer et al., 1994) and stress management (Casey, 2013). Past research has also demonstrated that this form of music use is associated with the tendency to use positive and adaptive emotion regulation strategies (Chin and Rickard, 2012b; Saarikallio, 2008). In addition, music use for the purposes of cognitive and emotional regulation consistently predicted the habitual use of cognitive reappraisal, and was more likely to predict higher levels of well-being through the use of cognitive reappraisal (Chin and Rickard, 2014). No significant direct link was reported between music use and any of the four aspects of well-being (subjective, psychological, emotional and social) measured and it was proposed that music engagement for cognitive and emotional regulation purposes may predict well-being primarily through the habitual use of cognitive reappraisal as an emotion regulation strategy (Chin and Rickard, 2014).

The capacity to regulate emotions is vital for psychosocial functioning (Gross, 2007; Gross and Thompson, 2007; Nyklíček et al., 2011) and differential associations have been reported between usage of various types of emotion regulation strategies and important outcomes such as social functioning (Eisenberg et al., 2000), physical and mental health (Gross and John, 2003; Gross and Muñoz, 1995; Sapolsky, 2007). There are several definitions of emotion regulation (Campos et al., 2004; Koole, 2009). This study adopts the definition of emotion regulation as the processes that individuals use to manage their emotional experience and expression, particularly focusing on two regulation processes within the process model of emotion regulation (Gross, 2007): cognitive change and response modulation. These two processes are most pertinent to the use of music to regulate emotions as they occur in everyday life, and more importantly, these two processes are differentially related to mental health outcomes. The capacity to use cognitive reappraisal, a cognitive change strategy, is linked with greater positive affect, higher levels of well-being, diminished negative affect and lower levels of psychopathology symptoms (Aldao et al., 2010; Gross and John, 2003; Haga et al., 2009; John and Gross, 2004). In contrast, the habitual use of expressive suppression, a response-focused strategy, is linked with lower levels of positive affect and well-being, as well as elevated levels of negative affect and psychopathology symptoms (Aldao et al., 2010; Gross and John, 2003; John and Gross, 2004). It is not surprising then that well-being outcomes of active music engagement are mediated by the type of emotion regulation strategy used. What remains unclear, however, is whether the positive mental health outcomes are sustained even after controlling for levels of positive and negative affective styles.

\section{Beyond positive and negative trait affect}

Trait affect represents stable individual predispositions to certain affective states. Trait positive affect refers to the disposition to experience positive feelings such as joy, contentment, and enthusiasm (Clark et al., 1989), while trait negative affect refers to the disposition to experience negative feelings such as anger, distress, and nervousness (Watson and Clark, 1984). Importantly, while individuals with higher trait negative affect are more likely to experience negative affective states, the disposition to negative affect does not necessarily imply a lack of positive affect. Just as individuals with high 
trait negative affect may experience both positive and negative affective states, individuals with high trait positive affect may also experience both affective states (Watson and Clark, 1984). More recently, trait affect has been demonstrated to predict change in the use of emotion regulation strategies, with negative affect predicting a significant increase, and positive affect predicting a significant reduction, in the use of emotion regulation strategies (Brans et al., 2013). Given that positive and negative affective states are used as indicators of subjective well-being, and have respectively been linked to positive and negative mental health outcomes (Brown et al., 1997; Fredrickson and Joiner, 2002; Lyubomirsky et al., 2005; Watson et al., 1995), trait affect should be controlled if the contribution of behavioural strategies (such as music use) to mental health outcomes is of interest. In particular, when investigating the unique contribution of music use on positive mental health, trait positive affect should be included as a covariate, to control for any benefits that are conferred by the disposition to experience positive feelings or moods. Likewise, trait negative affect should be included as a covariate, when measuring levels of depressive, anxiety and stress symptoms, to control for predisposition to experience negative feelings which may contribute to the increased use of emotion regulation strategies and may potentially mask the actual impact of emotion regulation on the relationship between music use and negative emotional states.

The current study aimed to examine the mediating role of emotion regulation on the relation between music use for cognitive and emotional regulation and mental health outcomes whilst controlling for trait affect. It was hypothesised that music use for cognitive and emotional regulation would predict positive mental health outcomes, as indicated by greater levels of well-being and lower levels of depressive, anxiety and stress symptoms, via the use of cognitive reappraisal as an emotion regulation strategy, even after controlling for trait affect. It was also anticipated that music use would predict poorer mental health outcomes, through the use of expressive suppression, even after controlling for trait affect.

\section{Method}

\section{Participants}

Five hundred and sixty-five participants (439 female, 126 male) between the ages of 18 and 68 years $(M=24.00, S D=6.21)$ provided consent and completed the survey for this study. Participants were recruited via posters and word-of-mouth through staff and students of Monash University. The majority of participants reported that they had played a musical instrument (73.45\%), and of these 415 participants, slightly over a third of participants $(n=156)$ are still currently playing an instrument. About two thirds of the sample indicated that they listen to music for at least one to two hours per day $(66.73 \%)$. Nearly a fifth of the participants $(n=105)$ indicated more than five hours of intentional music listening each day.

\section{Materials}

\section{Music engagement for the purposes of cognitive and emotional regulation}

The use of music for cognitive and emotional regulation purposes was measured using the 32-item Music Use questionnaire (MUSE; Chin and Rickard, 2012b). The Cognitive and Emotional Regulation subscale has seven items $(\alpha=.83)$, and a sample item is "I use a particular type of music to get me through tough times". Participants 
rated each item on a 6-point Likert-type scale, ranging from 0 (not at all/not applicable to me) to 5 (strongly agree). Alpha coefficient for this subscale was .75 in the current study.

\section{Emotion regulation strategies}

The 10-item Emotion Regulation Questionnaire (ERQ; Gross and John, 2003) was used to measure an individual's tendency to use reappraisal and suppression strategies for emotion regulation. The Cognitive Reappraisal subscale has six items $(\alpha=.79)$, and a sample item is "When I want to feel less negative emotion, I change the way I'm thinking about the situation". The Expressive Suppression subscale has four items $(\alpha=.73)$, and a sample item is "I control my emotions by not expressing them". Participants rated each item on a 7-point Likert-type scale, ranging from 1 (strongly disagree) to 7 (strongly agree). In the current study, alpha for the two subscales was .85 and .77 respectively.

\section{Mental health}

The Mental Health Continuum-Short Form (MHC-SF; Keyes et al., 2008) consists of three subscales measuring the level of an individual's well-being in each of the following domains: emotional well-being (3-item), social well-being (5-item), and psychological well-being (6-item), each demonstrating sound psychometric properties, with alpha of .83, .74, and .83, respectively (Lamers et al., 2011). Participants indicated the extent to which they experienced each aspect of well-being during the past month using a 6-point scale, from 1 (never) to 6 (every day). In the current study, alpha of the three subscales were $.90, .85$, and .89 , respectively.

\section{Negative emotional states of depression, anxiety and stress}

The 21-item Depression Anxiety Stress Scale (DASS21; Lovibond and Lovibond, 1995) was used to measure the presence of negative emotional states of depression, anxiety and stress. The depression subscale measures hopelessness, anhedonia, lack of interest, and a sample item is "I felt that life was meaningless". The anxiety subscale is an indication of one's subjective experience of autonomic arousal and anxious affect, and a sample item is "I felt I was close to panic". The stress subscale assesses levels of chronic non-specific arousal such as being easily agitated or irritable, and having difficulty relaxing, and a sample item is "I found it difficult to relax". For each item, participants were asked to indicate the degree to which the statement applied to them over the past week, on a scale of 0 (Did not apply to me at all) to 3 (Applied to me very much, or most of the time). Scores of each of the subscales were calculated by summing the scores of the seven items in each subscale and multiplied by two to obtain comparable scores to the published norms for the DASS. The DASS has been shown to have high internal consistency and temporal reliability (Lovibond and Lovibond, 1995), as well as good convergent and discriminant validity (Antony et al., 1998). In the current study, alpha coefficients for the depression, anxiety and stress subscales were .88 , .80 , and .84 , respectively. 


\section{Positive and negative trait affect}

Trait affect was measured using the 10-item International Positive and Negative Affect Schedule Short-Form (I-PANAS-SF; Thompson, 2007) with five items measuring positive affect (PA) and five items measuring negative affect (NA). Participants rated the extent to which they generally experience each of the 10 emotions on a 5-point Likert-type scale, ranging from 1 (never) to 5 (always). Scores for each subscale were obtained by summing responses from the respective items, with higher scores indicating greater levels of positive affect $(\alpha=.80)$ or negative affect $(\alpha=.74)$. In the current study, alpha for the positive and negative affect subscales were .81 , and .76, respectively.

\section{Procedure}

The questionnaires were made available online via SurveyMethods software (www.surveymethods.com). After agreeing to participate in the study, participants were provided with the link to the online survey, where they provided informed consent and completed the survey. All procedures were approved by Monash University Human Research Ethics Committee.

\section{Results}

Data were handled and analyzed using IBM SPSS version 21. Participants' response data and timings were screened for non-serious responding (as recommended by Reips, 2002). The standardized distribution of dependent variables was examined in order to identify and remedy any potential univariate outliers, and no outliers (+/- $2.96 S D s)$ were found. Variables were also examined for skewness and kurtosis, and all were found to be normally distributed, and multivariate assumptions of independence and normally distributed errors were met. Finally multicollinearity was assessed among the predictor variables, and no violations were identified.

\section{Descriptive analyses}

This sample consisted of individuals with mostly moderate ( $48 \%$ of the sample) to flourishing ( $47 \%$ of the sample) mental health scores and a small proportion with languishing (5\% of the sample) mental health. The majority of participants were within the normal score range for depression (57\% of the sample), anxiety ( $47 \%$ of the sample) and stress (61\% of the sample). Nearly a third of individuals indicated that they experienced mild to moderate symptoms (depression: 29\%; anxiety: 31\%; stress: 29\%), with the remaining proportion of participants reporting a greater severity of symptoms (depression: $14 \%$; anxiety: $22 \%$; stress: $10 \%$ ).

Pearson correlations, means, standard deviations and range of scores of the variables are presented in Table 1.

Music use for the purposes of cognitive and emotional regulation was positively associated with the use of the antecedent-focused strategy of cognitive reappraisal and the negative emotional state of stress.

Cognitive reappraisal was positively associated with the global measure of wellbeing and negatively correlated with all three measures of negative emotional states. In contrast, expressive suppression was negatively associated with the global 
Table 1 Means, standard deviations, range of scores and intercorrelations between key variables

\begin{tabular}{|c|c|c|c|c|c|c|c|c|c|c|c|c|}
\hline & & $M$ & $S D$ & Range & 1 & 2 & 3 & 4 & 5 & 6 & 7 & 8 \\
\hline 1 & $\begin{array}{l}\text { Music use for cognitive } \\
\text { and emotional regulation }\end{array}$ & 34.90 & 4.18 & $20-42$ & - & & & & & & & \\
\hline 2 & ER-Reappraisal & 29.45 & 5.90 & $6-42$ & $.15^{* *}$ & - & & & & & & \\
\hline 3 & ER-Suppression & 15.20 & 4.90 & $4-28$ & .07 & $.15^{* *}$ & - & & & & & \\
\hline 4 & Well-being & 45.81 & 13.64 & $0-70$ & .04 & $.37^{* *}$ & $-.21^{* *}$ & - & & & & \\
\hline 5 & Depression & 10.13 & 9.18 & $0-42$ & .07 & $-.28^{* *}$ & $.25^{* *}$ & $-.52^{* *}$ & - & & & \\
\hline 6 & Anxiety & 9.51 & 8.14 & $0-42$ & .07 & $-.17^{* *}$ & $.22^{* *}$ & $-.30^{* *}$ & $.64^{* *}$ & - & & \\
\hline 7 & Stress & 13.66 & 8.76 & $0-42$ & $.12^{* *}$ & $-.26^{* *}$ & $.11^{* *}$ & $-.37^{* *}$ & $.71^{* *}$ & $.70^{* *}$ & - & \\
\hline 8 & Positive affect & 20.77 & 4.71 & $5-25$ & $.16^{* *}$ & $.31^{* *}$ & $-.11^{* *}$ & $.48^{* *}$ & $-.31^{* *}$ & $-.14^{* *}$ & $-.14^{* *}$ & - \\
\hline 9 & Negative affect & 14.22 & 5.11 & $5-25$ & $.15^{* *}$ & $-.18^{* *}$ & $.11^{*}$ & $-.28^{* *}$ & $.47^{* *}$ & $.45^{* *}$ & $.55^{* *}$ & -.07 \\
\hline
\end{tabular}

measure of well-being and positively correlated with the measures of depression, anxiety, and stress.

Positive affect was positively correlated with music use for cognitive and emotional regulation, cognitive reappraisal and well-being, and therefore included as a covariate in the mediation analysis of well-being. Negative affect was also significantly associated with the key variables and hence included as a covariate in the mediation analyses of depression, anxiety and stress.

\section{Test of mediation}

Bootstrap resampling methods (Preacher and Hayes, 2004, 2008) were conducted for each mediation analysis (see Figure 1) to assess the indirect effect of the independent variable (music engagement) on the dependent variable (mental health outcome) via

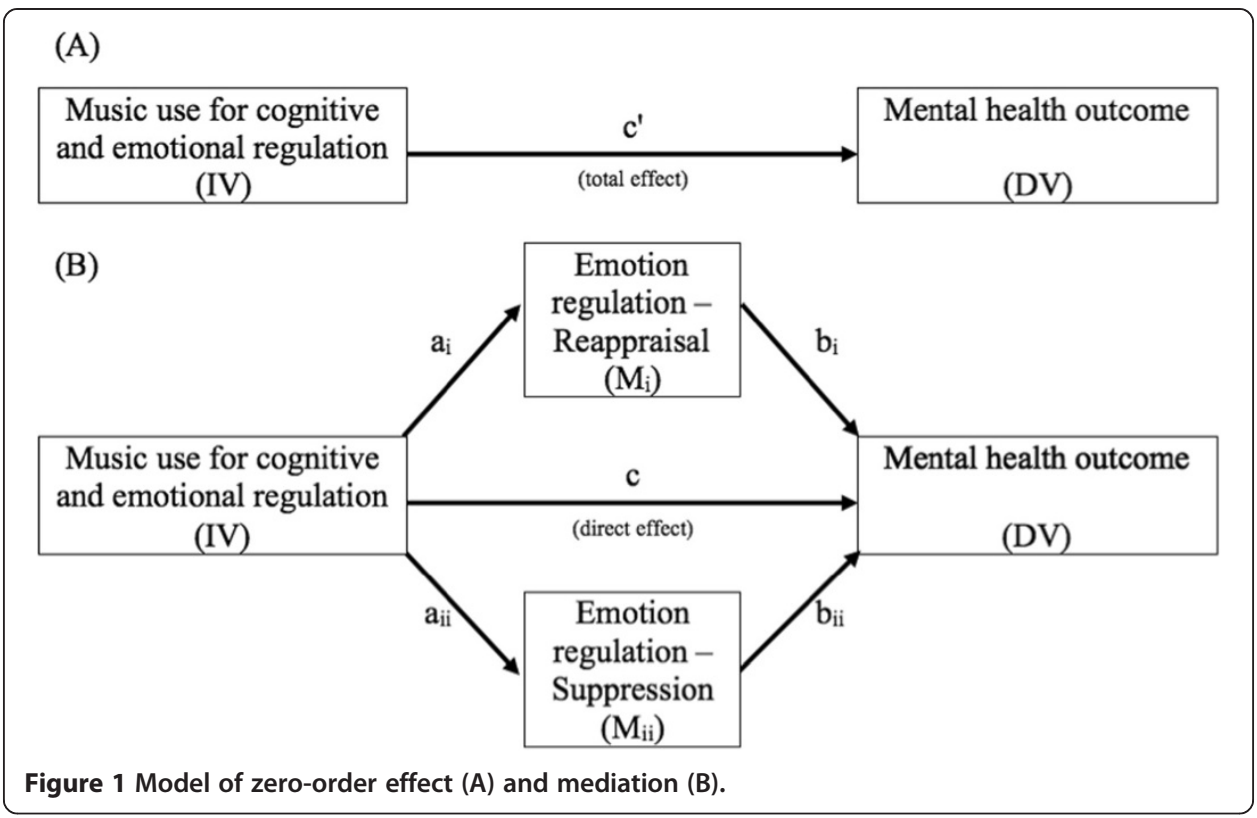


the mediator (emotion regulation). Positive affect was included as a covariate in the mediation analysis of well-being, and negative affect was included as a covariate in the mediation analyses of depression, anxiety and stress.

The bootstrap method of examining mediation models is increasingly recommended over the traditional Baron and Kenny analyses of mediation or use of the Sobel test (MacKinnon et al., 2002), as it allows for unexpected direct effects and potential omitted indirect paths of the same sign as the direct effect, and uses the more rigorous and powerful bootstrap test in examining the indirect effect (Zhao et al., 2010). Bootstrapping is particularly advantageous when applied to multiple mediation (such as required for emotion regulation strategies) as it allows estimation of individual indirect effects and contrasts among indirect effects without potential problems due to collinearity that may pose a problem in path analysis (Preacher and Hayes, 2008). As per Preacher and Hayes (2008) recommendations, 10,000 resamples were generated to create biascorrected $95 \%$ confidence intervals (CI). CIs which do not include a zero value indicate a significant indirect effect. The results obtained from the four analyses are summarized in Table 2.

Indirect-only mediation through cognitive reappraisal was observed between music use for cognitive and emotional regulation and measures of mental health outcome. Despite not having a significant direct effect on predicting improved mental health outcomes, music use for cognitive and emotional regulation predicted better well-

Table 2 Path coefficients, estimates and confidence intervals for mediation analyses of mental health outcomes

\begin{tabular}{|c|c|c|c|c|c|c|c|c|c|}
\hline & $\mathrm{IV} \rightarrow \mathrm{M}_{\mathrm{i}}$ & $\mathrm{M}_{\mathrm{i}} \rightarrow \mathrm{DV}$ & $\begin{array}{l}\text { Indirect } \\
\text { via } M_{i}\end{array}$ & $\begin{array}{l}\text { Direct } \\
\text { effect }\end{array}$ & $\begin{array}{l}\text { Total } \\
\text { effect }\end{array}$ & $\mathrm{IV} \rightarrow \mathrm{M}_{\mathrm{ii}}$ & $M_{i i} \rightarrow D V$ & $\begin{array}{l}\text { Indirect } \\
\text { via } M_{\mathrm{ii}}\end{array}$ & $\begin{array}{l}\text { Indirect effect } \\
\text { contrast }\end{array}$ \\
\hline & $a_{i}$ & $b_{i}$ & $a_{i} \times b_{i}$ & c & $c^{\prime}$ & $a_{i i}$ & $b_{i i}$ & $a_{i i} \times b_{i i}$ & $\left(a_{i} \times b_{i}\right)-\left(a_{i i} \times b_{i i}\right)$ \\
\hline \multicolumn{10}{|c|}{ Well-being } \\
\hline B & $.1526^{* *}$ & $.6735^{* *}$ & .1029 & -.1011 & -.1404 & $.1101^{*}$ & $-.5762^{* *}$ & -.0624 & .1653 \\
\hline$S E$ & .0569 & .0864 & .0449 & .1222 & .1155 & .0495 & .0992 & .0310 & .0602 \\
\hline $\mathrm{LLCl}$ & & & .0256 & & & & & -.1321 & .0611 \\
\hline ULCI & & & .2032 & & & & & -.0089 & .3018 \\
\hline \multicolumn{10}{|c|}{ Depression } \\
\hline$B$ & $.2588^{* *}$ & $-.4007^{* *}$ & -.1044 & -.0021 & .0693 & .0699 & $.4626^{* *}$ & .0324 & -.1368 \\
\hline SE & .0581 & .0570 & .0340 & .0829 & .0788 & .0496 & .0667 & .0250 & .0468 \\
\hline $\mathrm{LLCl}$ & & & -.1833 & & & & & -.0127 & -.2376 \\
\hline ULCI & & & -.0486 & & & & & .0861 & -.0526 \\
\hline \multicolumn{10}{|c|}{ Anxiety } \\
\hline$B$ & $.2588^{* *}$ & $-.1799^{* *}$ & -.0469 & .0098 & .0337 & .0699 & $.3248^{* *}$ & .0233 & -.0701 \\
\hline$S E$ & .0581 & .0531 & .0214 & .0742 & .0734 & .0496 & .0622 & .0182 & .0304 \\
\hline $\mathrm{LLCl}$ & & & -.1037 & & & & & -.0085 & -.1394 \\
\hline ULCI & & & -.0146 & & & & & .0645 & -.0192 \\
\hline \multicolumn{10}{|c|}{ Stress } \\
\hline$B$ & $.2588^{* *}$ & $-.2829^{* *}$ & -.0741 & .0772 & .1401 & .0699 & $.1476^{*}$ & .0103 & -.0844 \\
\hline$S E$ & .0581 & .0534 & .0257 & .0744 & .0739 & .0496 & .0625 & .0098 & .0290 \\
\hline $\mathrm{LLCl}$ & & & -.1350 & & & & & -.0021 & -.1523 \\
\hline ULCI & & & -.0331 & & & & & .0389 & -.0374 \\
\hline
\end{tabular}

${ }^{* *} p<.01 ; * p<.05$.

Value in bold indicates statistical significance on $95 \%$ confidence interval using bootstrapping with 10,000 samples. 
being and lower symptoms of depression, anxiety and stress, through the emotion regulation strategy of cognitive reappraisal. Music use also predicted the tendency to use suppression, which in turn predicted lower well-being ratings. This negative indirect-only mediation through suppression is significantly weaker than the positive indirect-only mediation through reappraisal, as indicated by the significantly positive indirect effect contrast. Similarly for depression, anxiety and stress ratings, the strength of the mediation pathway via reappraisal is significantly stronger than suppression.

\section{Discussion}

This study provides initial evidence that music use for cognitive and emotional regulation, independent of an individual's affective style, may be beneficial for mental health. Positive outcomes are mainly predicted by the use of cognitive reappraisal, rather than the use of suppressive emotion regulation strategies. We hypothesised that music use for cognitive and emotional regulation may predict higher levels of well-being and reduced symptoms of depression, anxiety and stress, through cognitive reappraisal, even after controlling for trait affect. In support, we found that intentional use of music for the purposes of cognitive and emotional regulation, independent of affect, consistently predicted positive mental health outcomes through the use of antecedent-focused emotion regulation strategy of cognitive reappraisal. In contrast, music engagement for cognitive and emotional regulatory purposes was more likely to predict poorer well-being through the use of expressive suppression, a response-focused strategy.

Earlier studies examining the role of emotion regulation in the relationship between music engagement and well-being outcomes have demonstrated that it is the way music is used that predicts positive well-being outcomes, rather than mere exposure to music (Chin and Rickard, 2014). Extending on this earlier work, trait affect was included as a covariate in the current study. Mental health is not merely the presence and absence of emotional states, but the presence of positive psychological, emotional and social functioning (Keyes, 2002). Individuals with high trait positive affect are more likely to experience positive feelings and moods, and also seek out activities which may facilitate or maintain positive functioning, which in turn contribute to enhanced overall well-being. Music is one such example, amongst other activities such as physical exercise, mindfulness or meditation. It is then not sufficient to simply demonstrate that a particular activity predicts or contributes to positive mental health as this could simply reflect or result from high positive affective styles. To clearly demonstrate that positive mental health may be achieved by active engagement with an activity, music use for cognitive and emotional regulation in this case, trait positive affect should be statistically controlled for. Findings here demonstrate that intentional music use for cognitive and emotional regulation may predict positive mental health outcomes, even after controlling for trait positive affect. This suggests that observed beneficial mental health outcomes were uniquely accounted for by music use for cognitive and emotional purposes, through the use of cognitive reappraisal emotion regulation strategy, and not the disposition to experience positive feelings and moods. In addition, Brans et al. (2013) found that high levels of positive affect predicted lesser use of cognitive reappraisal as an emotion regulation strategy. By including trait positive affect as a covariate in the current mediation analysis, this 
allowed us to clearly distinguish unique contributions of music use for cognitive and emotional regulation on both cognitive reappraisal as an emotion regulation style and mental health outcomes, from the potential influence of trait positive affect. This lends greater support to the proposition that a high level of music engagement predicts greater use of cognitive reappraisal as an emotion regulation style, which in turn predicts positive mental health outcomes, rather than music engagement being an adaptive outcome of good mental health. Further research utilizing event and experience sampling may provide more insight by measuring trait affect in the natural contexts and situations of everyday life.

Even though negative affect is adaptive for survival, a chronic state of distress is strongly linked to depression, anxiety and stress (Brown et al., 1997; Watson et al., 1988). Research has demonstrated the efficacy of music use to induce positive affective states (North et al., 2004) and achieve desired moods (Västfjäll, 2002). In this sample, the tendency to engage with music for the purposes of cognitive and emotional regulation was associated with higher levels of trait negative affect and stress symptoms. These results were consistent with past research that reported the frequent use of music to enhance mood (Juslin and Laukka, 2004; North et al., 2000) and cope with symptoms of stress (Bradt and Dileo, 2009). Despite finding that greater negative affect predicted increased use of emotion regulation strategies, Brans et al. (2013) also reported that use of adaptive strategies such as reappraisal did not predict subsequent change in negative affect. The latter finding is in contrast to several other reports, which have linked the use of cognitive reappraisal with diminished negative affect and lower levels of psychopathology symptoms (Aldao et al., 2010; Gross and John, 2003; Haga et al., 2009; John and Gross, 2004). Findings here may offer new insights into this discrepancy. Trait negative affect refers to the disposition to experience negative feelings and states. Individuals with higher levels of trait negative affect are more vulnerable to symptoms of depression, anxiety and stress. However, there remains a possibility that the links between trait negative affect and symptoms of mental illness could reflect subjective biases in symptom reporting (Cohen et al., 1995). As with mental health outcomes, measurements of negative emotional states such as depression, anxiety and stress should statistically control for levels of trait negative affect. This removes the subjective biases in symptom reporting and allows differentiation of the experience of negative emotional states from the trait disposition towards negative affective styles. By including trait negative affect as a covariate in the current mediation analyses, the significant prediction of fewer depressive, anxiety and stress symptoms could be attributed to the use of cognitive reappraisal as an emotion regulation strategy. Furthermore, this was not observed for expressive suppression. This clearly suggests that individuals using music for cognitive and emotional regulation, independent of disposition towards experiencing negative affect, are more likely to experience fewer symptoms of depression, anxiety and stress, if they habitually reappraise events to modify subjective feelings, rather than suppressing or avoiding the feelings. Therefore, the use of music for cognitive and emotional regulation could be a particularly viable method to develop the habitual use of adaptive emotion regulation strategies that promote flourishing in everyday life, regardless of one's trait disposition towards negative affect. These findings also emphasize the need to reassess how music is recommended as a selfregulatory tool.

This study relied exclusively on self-reports to assess mental health outcomes, level of music engagement and the type of emotion regulation strategy used. Despite drawbacks 
such as reporting biases and social desirability motivations (Scollon et al., 2003), self-reports provide a cost-effective way to collect survey responses from a large number of individuals, from more than one geographical location. Efforts were made to recruit a large sample of participants, from diverse cultural, socioeconomic and musical backgrounds. However, the majority of the sample was female and this may limit the generalizability of the findings.

This study also focused on only one antecedent-focused emotion regulation strategy, cognitive reappraisal, and one response-focused strategy of expressive suppression. While these strategies are the most commonly researched in the literature (Gross and John, 2003; Hofmann et al., 2009), future research will need to investigate if the current findings also apply to other types of regulation strategies. For instance, another type of emotion regulation strategy in the process model that individuals may use is attentional deployment. Individuals may strategically choose to engage with music activities to direct their attention away from situations or stimuli that provoke undesirable emotions. By directing their attention to the music activity, individuals may be able to reduce the impact of the emotion generated. It would also be useful to examine the stability of mental health outcomes of individuals using music in the various stages of the process model over time.

An important consideration here is that statistical mediation does not establish causal direction. This method allowed for the inclusion of a multicategorical mediator like emotion regulation, as well as covariates of positive and negative trait affect. Insights were obtained about the pathways in which music use is beneficial or detrimental for mental health. Despite deriving the proposed mediation model from past research, the current findings do not preclude other explanations. For example, individuals with adaptive emotion regulation strategies may already use music effectively to cope with undesirable thoughts and emotions or actively seek methods to facilitate self-regulation. Therefore, the sequence of mediation effects reported here will require further exploration in future research.

To conclude, findings here show that individuals highly engaged with music for the purposes of cognitive and emotional regulation are more likely to use cognitive reappraisal as an emotion regulation strategy, and this in turn predicts positive mental health outcomes. Importantly, the prospective benefits of music use for cognitive and emotional regulation are sustained even after controlling for positive and negative trait affect, in the outcome measures of mental health and negative emotional states, respectively. This suggests that purposeful music use has the potential to elevate well-being indices beyond levels typically linked to positive trait affect, and lessen depressive, anxiety and stress symptoms from levels typically associated with negative trait affect. This study provides new evidence that purposeful music engagement may promote flourishing in life, by alleviating mental distress and enhancing well-being, through cognitive reappraisal, even after controlling for trait affect.

Competing interests

The authors declare that they have no competing interests.

Authors' contributions

Both TC and NR contributed to the design and coordination of the study. TC performed the statistical analysis and drafted the manuscript. NR helped to revise the manuscript. All authors read and approved the final manuscript.

Author details

${ }^{1}$ Melbourne Graduate School of Education, The University of Melbourne, 100 Leicester Street, Parkville (3010), VIC, Australia. ${ }^{2}$ School of Psychological Sciences, Monash University, Wellington Road, Clayton (3800), VIC, Australia. 
Received: 21 May 2014 Accepted: 13 November 2014

Published online: 18 December 2014

\section{References}

Aldao, A, Nolen-Hoeksema, S, \& Schweizer, S. (2010). Emotion regulation strategies across psychopathology: a meta-analytic review. Clinical Psychology Review, 30, 217-237.

Antaramian, SP, Huebner, ES, Hills, KJ, \& Valois, RF. (2010). A dual-factor model of mental health: toward a more comprehensive understanding of youth functioning. American Journal of Orthopsychiatn, 80, 462-472.

Antony, MM, Bieling, PJ, Cox, BJ, Enns, MW, \& Swinson, RP. (1998). Psychometric properties of the 42-item and 21-item versions of the Depression Anxiety Stress Scales (DASS) in clinical groups and a community sample. Psychological Assessment, 10, 176-181.

Bishop, DT, Karageorghis, Cl, \& Loizou, G. (2007). A grounded theory of young tennis players' use of music to manipulate emotional state. Journal of Sport \& Exercise Psychology, 29, 584-607.

Bradt, J, \& Dileo, C. (2009). Music for stress and anxiety reduction in coronary heart disease patients. Cochrane Database of Systematic Reviews, (2), doi: 10.1002/14651858.CD006577.pub2.

Bradt, J, Dileo, C, Grocke, D, \& Magill, L. (2011). Music interventions for improving psychological and physical outcomes in cancer patients. Cochrane Database of Systematic Reviews, (8), doi: 10.1002/14651858.CD006911.pub2.

Brans, K, Koval, P, Verduyn, P, Lim, YL, \& Kuppens, P. (2013). The regulation of negative and positive affect in daily life. Emotion, 13, 926-939.

Brown, TA, Chorpita, BF, Korotitsch, W, \& Barlow, DH. (1997). Psychometric properties of the Depression Anxiety Stress Scales (DASS) in clinical samples. Behaviour Research and Therapy, 35, 79-89.

Campos, JJ, Frankel, CB, \& Camras, L. (2004). On the nature of emotion regulation. Child Development, 75, 377-394.

Casey, L. (2013). Stress and wellbeing in Australia survey 2013. Melbourne: The Australian Psychological Society.

Chamorro-Premuzic, T, \& Furnham, A. (2007). Personality and music: can traits explain how people use music in everyday life? British Journal of Psychology, 98, 175-185.

Chin, TC, \& Rickard, NS. (2012a). Re-conceptualizing 'musicianship': Music performance and training through to music reception and engagement. In NS Rickard \& K McFerran (Eds.), Lifelong engagement with music: Benefits for mental health and well-being (pp. 161-180). New York: Nova Science Publishers, Inc.

Chin, TC, \& Rickard, NS. (2012b). The Music USE (MUSE) questionnaire: an instrument to measure engagement in music. Music Perception, 29, 429-446.

Chin, TC, \& Rickard, NS. (2014). Emotion regulation strategy mediates both positive and negative relationships between music uses and well-being. Psychology of Music, 42, 692-713.

Clark, LA, Watson, D, \& Leeka, J. (1989). Diurnal variation in the positive affects. Motivation and Emotion, 13, 205-234.

Cohen, S, Doyle, WJ, Skoner, DP, Fireman, P, Gwaltney, JM, Jr, \& Newman, JT. (1995). State and trait negative affect as predictors of objective and subjective symptoms of respiratory viral infections. Journal of Personality and Social Psychology, 68, 159-169.

DeNora, T. (1999). Music as a technology of the self. Poetics, 27, 31-56

DeNora, T. (2000). Music in everyday life. Cambridge: Cambridge University Press.

Eisenberg, N, Fabes, RA, Guthrie, IK, \& Reiser, M. (2000). Dispositional emotionality and regulation: their role in predicting quality of social functioning. Journal of Personality and Social Psychology, 78, 136-157.

Fredrickson, BL, \& Joiner, T. (2002). Positive emotions trigger upward spirals toward emotional well-being. Psychological Science, 13, 172-175.

Gross, JJ. (2007). Handbook of emotion regulation. New York, NY: Guilford Press.

Gross, JJ, \& John, OP. (2003). Individual differences in two emotion regulation processes: implications for affect, relationships, and well-being. Journal of Personality and Social Psychology, 85, 348-362.

Gross, JJ, \& Muñoz, RF. (1995). Emotion regulation and mental health. Clinical Psychology Science \& Practice, 2, 151-164.

Gross, JJ, \& Thompson, R. (2007). Emotion regulation: Conceptual foundations. In JJ Gross (Ed.), Handbook of emotion regulation (pp. 3-24). New York: Guilford Press.

Haga, SM, Kraft, P, \& Corby, EK. (2009). Emotion regulation: antecedents and well-being outcomes of cognitive reappraisal and expressive suppression in cross-cultural samples. Journal of Happiness Studies, 10, 271-291.

Hargreaves, DJ, \& North, AC. (1997). The social psychology of music. Oxford: Oxford University Press.

Hays, T, \& Minichiello, V. (2005). The meaning of music in the lives of older people: a qualitative study. Psychology of Music, 33, 437-451.

Hofmann, SG, Heering, S, Sawyer, AT, \& Asnaani, A. (2009). How to handle anxiety: the effects of reappraisal, acceptance, and suppression strategies on anxious arousal. Behaviour Research and Therapy, 47, 389-394.

John, OP, \& Gross, JJ. (2004). Healthy and unhealthy emotion regulation: personality processes, individual differences, and life span development. Journal of Personality, 72, 1301-1333.

Juslin, PN, \& Laukka, P. (2004). Expression, perception, and induction of musical emotions: a review and a questionnaire study of everyday listening. Journal of New Music Research, 33, 217-238,

Karageorghis, Cl, \& Terry, PC. (1997). The psychophysical effects of music in sport and exercise: a review. Journal of Sport Behavior, 20, 54-68.

Keyes, CLM. (2002). The mental health continuum: from languishing to flourishing in life. Journal of Health and Social Behavior, 43, 207-222.

Keyes, CLM. (2005). Mental illness and/or mental health? investigating axioms of the complete state model of health. Journal of Consulting and Clinical Psychology, 73, 539-548.

Keyes, CLM. (2006). Mental health in adolescence: is America's youth flourishing? American Journal of Orthopsychiatry, 76, 395-402.

Keyes, CLM, Wissing, M, Potgieter, JP, Temane, M, Kruger, A, \& van Rooy, S. (2008). Evaluation of the Mental Health Continuum Short Form (MHC-SF) in Setswana speaking South Africans. Clinical Psychology and Psychotherapy, 15, 181-192.

Keyes, CLM, Dhingra, SS, \& Simoes, EJ. (2010). Change in level of positive mental health as a predictor of future risk of mental illness. American Journal of Public Health, 100, 2366-2371. 
Koole, SL. (2009). The psychology of emotion regulation: an integrative review. Cognition and Emotion, 23, 4-41. Laiho, S. (2004). The psychological functions of music in adolescence. Nordic Journal of Music Therapy, 13, 49-65. Lamers, SMA, Westerhof, GJ, Bohlmeijer, ET, Klooster, PM, \& Keyes, CLM. (2011). Evaluating the psychometric properties of the Mental Health Continuum-Short Form (MHC-SF) in the Dutch Population. Journal of Clinical Psychology, 67, 99-110. Laukka, P. (2007). Uses of music and psychological well-being among the elderly. Journal of Happiness Studies, 8, 215-241. Lesiuk, T. (2005). The effect of music listening on work performance. Psychology of Music, 33, 173-191.

Lovibond, SH, \& Lovibond, PF. (1995). Manual for the depression anxiety stress scales (2nd ed.). Sydney: Psychology Foundation. Lyubomirsky, S, King, LA, \& Diener, E. (2005). The benefits of frequent positive affect: does happiness lead to success? Psychological Bulletin, 131, 803-851.

MacDonald, RAR, Hargreaves, DJ, \& Miell, DE (Eds.). (2002). Musical identities. Oxford: Oxford University Press MacDonald, R, Kreutz, G, \& Mitchell, L. (2012). Music, health and wellbeing. New York: Oxford University Press Inc. Mackinnon, DP, Lockwood, CM, Hoffman, JM, West, SG, \& Sheets, V. (2002). A comparison of methods to test the significance of the mediated effect. Psychological Methods, 7, 83-104.

Maratos, A, Gold, C, Wang, X, \& Crawford, M. (2008). Music therapy for depression. Cochrane Database of Systematic Reviews, (1), doi: 10.1002/14651858.CD004517.pub2.

Miranda, D, \& Claes, M. (2007). Musical preferences and depression in adolescence. International Journal of Adolescence and Youth, 13, 285-309.

Miranda, D, \& Claes, M. (2009). Music listening, coping, peer affiliation and depression in adolescence. Psychology of Music, 37, 215-233.

North, AC, \& Hargreaves, DJ. (2006). Problem music and self-harming. Suicide and Life-Threatening Behavior, 36, 582-590.

North, AC, \& Hargreaves, DJ (Eds.). (2008). The social and applied psychology of music. Oxford: Oxford University Press.

North, AC, Hargreaves, DJ, \& O'Neill, SA. (2000). The importance of music to adolescents. British Journal of Educational Psychology, 70, 255-272.

North, AC, Hargreaves, DJ, \& Hargreaves, JJ. (2004). The uses of music in everyday life. Music Perception, 22, 63-99.

Nykliček, I, Vingerhoets, AJJM, \& Zeelenberg, M. (2011). Emotion regulation and well-being. New York, NY: Springer.

Preacher, KJ, \& Hayes, AF. (2004). SPSS and SAS procedures for estimating indirect effects in simple mediation models. Behavior Research Methods, Instruments, and Computers, 36, 717-731.

Preacher, KJ, \& Hayes, AF. (2008). Asymptotic and resampling strategies for assessing and comparing indirect effects in multiple mediator models. Behavior Research Methods, 40, 879-891.

Reips, UD. (2002). Standards for internet-based experimenting. Experimental Psychology, 49, 243-256,

Rickard, NS, \& McFerran, K. (2012). Lifelong engagement with music: benefits for mental health and well-being. New York: Nova Science Publishers, Inc.

Saarikallio, S. (2007). Music as mood regulation in adolescence. University of Jyväskylä: Doctoral dissertation.

Saarikallio, S. (2008). Music in mood regulation: initial scale development. Musicae Scientiae, 12, 291-310.

Saarikallio, S. (2011). Music as emotional self-regulation throughout adulthood. Psychology of Music, 39, 307-327.

Saarikallio, S, \& Erkkilä, J. (2007). The role of music in adolescents' mood regulation. Psychology of Music, 35, 88-109.

Sapolsky, RM. (2007). Stress, stress-related disease, and emotion regulation. In JJ Gross (Ed.), Handbook of emotion regulation (pp. 606-615). New York, NY: Guilford Press.

Scollon, CN, Kim-Prieto, C, \& Diener, E. (2003). Experience sampling: promises and pitfalls, strengths, and weaknesses. Journal of Happiness Studies, 4, 5-34.

Siedliecki, S, \& Good, M. (2006). Effect of music on power, pain, depression and disability. Journal of Advanced Nursing, 54, 553-562

Sloboda, JA. (1999). Everyday uses of music listening: A preliminary study. In YI Suk Won (Ed.), Music, mind, and science (pp. 354-369). Seoul: Seoul National University Press.

Standley, JM. (1995). Music as a therapeutic intervention in medical and dental treatment: research and clinica applications. In T Wigram, B Saperstone, \& R West (Eds.), The art and science of music therapy: a handbook (pp. 3-22). Langhorne: Harwood.

Suldo, SM, \& Shaffer, EJ. (2008). Looking beyond psychopathology: the dual factor model of mental health in youth. School Psychology Review, 37, 52-68.

Thayer, RE, Newman, JR, \& McClain, TM. (1994). Self-regulation of mood: strategies for changing a bad mood, raising energy, and reducing tension. Journal of Personality and Social Psychology, 67, 910-925.

Thompson, ER. (2007). Development and validation of an internationally reliable short-form of the Positive and Negative Affect Schedule (PANAS). Journal of Cross-Cultural Psychology, 38, 227-242.

Västfjäll, D. (2002). Emotion induction through music: a review of the musical mood induction procedure. Musicae Scientiae, 6, 171-203.

Watson, D, \& Clark, LA. (1984). Negative affectivity: the disposition to experience aversive emotional states. Psychological Bulletin, 96, 465-490.

Watson, D, Clark, LA, \& Tellegen, A. (1988). Development and validation of brief measures of positive and negative affect: the PANAS scales. Journal of Personality and Social Psychology, 47, 1063-1070.

Watson, D, Weber, K, Assenheimer, JS, Clark, LA, Strauss, ME, \& McCormick, RA. (1995). Testing a tripartite model: . evaluating the convergent and discriminant validity of anxiety and depression symptom scales. Journal of Abnormal Psychology, 104, 3-14.

Westerhof, GJ, \& Keyes, CLM. (2010). Mental illness and mental health: the two continua model across the lifespan. Journal of Adult Development, 17, 110-119.

World Health Organization. (2005). Promoting mental health: Concepts, emerging evidence, practice. Geneva: WHO.

Zhao, X, Lynch, JG, \& Chen, Q. (2010). Reconsidering Baron and Kenny: myths and truths about mediation analysis. Journal of Consumer Research, 37, 197-206.

doi:10.1186/s13612-014-0025-4

Cite this article as: Chin and Rickard: Beyond positive and negative trait affect: Flourishing through music engagement. Psychology of Well-Being: Theory, Research and Practice 2014 4:25. 